\title{
Cryo-SEM and Raman Spectroscopy Study of the Involvement of Polyhydroxyalkanoates in Stress Response of Bacteria
}

\author{
V. Krzyzanek ${ }^{1}$, K. Hrubanova ${ }^{1}$, O. Samek ${ }^{1}$, S. Obruca ${ }^{2}$, I. Marova ${ }^{2}$, S. Bernatova ${ }^{1}$, M. Siler ${ }^{1}$, \\ P. Zemanek ${ }^{1}$ \\ 1. Institute of Scientific Instruments of the ASCR, v.v.i., Brno, Czech Republic. \\ 2. Centre for Material Research, Brno University of Technology, Brno, Czech Republic.
}

Many bacterial strains are capable of accumulation of very high amounts of polyhydroxyalkanoates (PHAs) in cytoplasm. Consequently, increasing content of the macromolecular material will strongly affect the overall material properties of cells and it is also likely that high content of PHAs inside the cells will also influence the rest of the cell volume - the cytoplasm. PHAs does not serve only as a carbon source in starvation, but presence of PHAs granules in cytoplasm represent additional advantage under stress conditions. It has an impact on metabolic activity of the cells and, moreover, it changes cells morphology as well as physico-chemical properties of the bacterial cytoplasm and cells.

The main aim of our investigations is to utilize combination of modern methods, such as electron and Raman microscopy [1] in the complex study on changes in physico-chemical properties of bacterial cells and cell cytoplasm with respect to intracellular content of PHA. In particular, we aim to study the influence of PHAs presence in the cell on its mechanical properties, mobility of solutes in cytoplasm, and thermal behavior. For this behavior of cells with various contents of PHAs is compared and referenced to the PHA none-producing mutant. For this purpose, we have utilized following bacterial strains Cupriavidus necator $\mathrm{H} 16$ and also its PHA none-producing mutant $C$. necator PHB. 'Further, we investigated Burholderia cepacia, Burholderia sacchari and Pseudomonas aeruginosa, which are capable of PHAs accumulation as well as biofilm formation.

For cryo scanning electron microscopy (cryo-SEM) a thin piece of sample was quickly frozen in liquid nitrogen, moved into a vacuum chamber (ACE600, Leica Microsystems) where it was freeze-fractured and shortly sublimated at $-95^{\circ} \mathrm{C}$. In the next step, the sample was moved at high vacuum using a shuttle (VCT100, Leica Microsystems) into the SEM (Magellan, FEI) equipped with a cold stage and the fractured structure was observed with $1 \mathrm{keV}$ electron beam at $-135^{\circ} \mathrm{C}$ without any metal coating. During freeze-fractured experiments on bacteria containing polyhydroxybutyrate (PHB) we observed interesting morphological features which showed needle/mushroom-type deformations (Fig.1a). Moreover, for the pure copolymer of 3-hydroxybutyrate and 3-hydroxyvalerate (P(HB-co-HV)) sample similar features were also observed (Fig.1b). This behavior was observed previously by Sudesh et al. [2] and explained by the heat which is generated during fracturing process which is considered to be sufficient to introduce the needle/mushroom-type morphology for PHA granules in the cells.

Raman micro-spectroscopic experiments with above mentioned cells were carried out using Renishaw system. Fig. 2 shows a typical Raman spectrum, here for Cupriavidus necator H16 cultured directly on a Petri dish. Raman spectroscopy seems to be very efficient tool for direct analysis of intracellular PHA content of bacteria and, furthermore, can be combined with SEM measurements to make it possible to measure nutrient dynamics and metabolism of the cells. Moreover, with this type of sensing one may detect variability of polymers contained within the cells which could help in elucidating different morphological features during the process of cryo-SEM technique. 
We believe that our study will be of significant assistance to research group being involved in bacterial strains which accumulate $\mathrm{PHB}$ and $\mathrm{P}(\mathrm{HB}-\mathrm{co}-\mathrm{HV})$ and for investigations of this material properties under low temperatures. Our results are convincing enough to warrant more extensive investigations with larger sets of bacterial strains to evaluate combination of cryo-SEM and Raman spectroscopy.

\section{References:}

[1] O. Samek et al, Sensors 10 (2010), p. 8635.

[2] K. Sudesh et al, Can J Microbiol 46 (2000), p. 304.

[3] This work received support from the Ministry of Health, Ministry of Education, Youth and Sports of the Czech Republic (LO1212), together with the European Commission (ALISI No. CZ.1.05/2.1.00/01.0017) and the Grant Agency of the Czech Republic (GACR GP15-20645S, GA14-20012S).
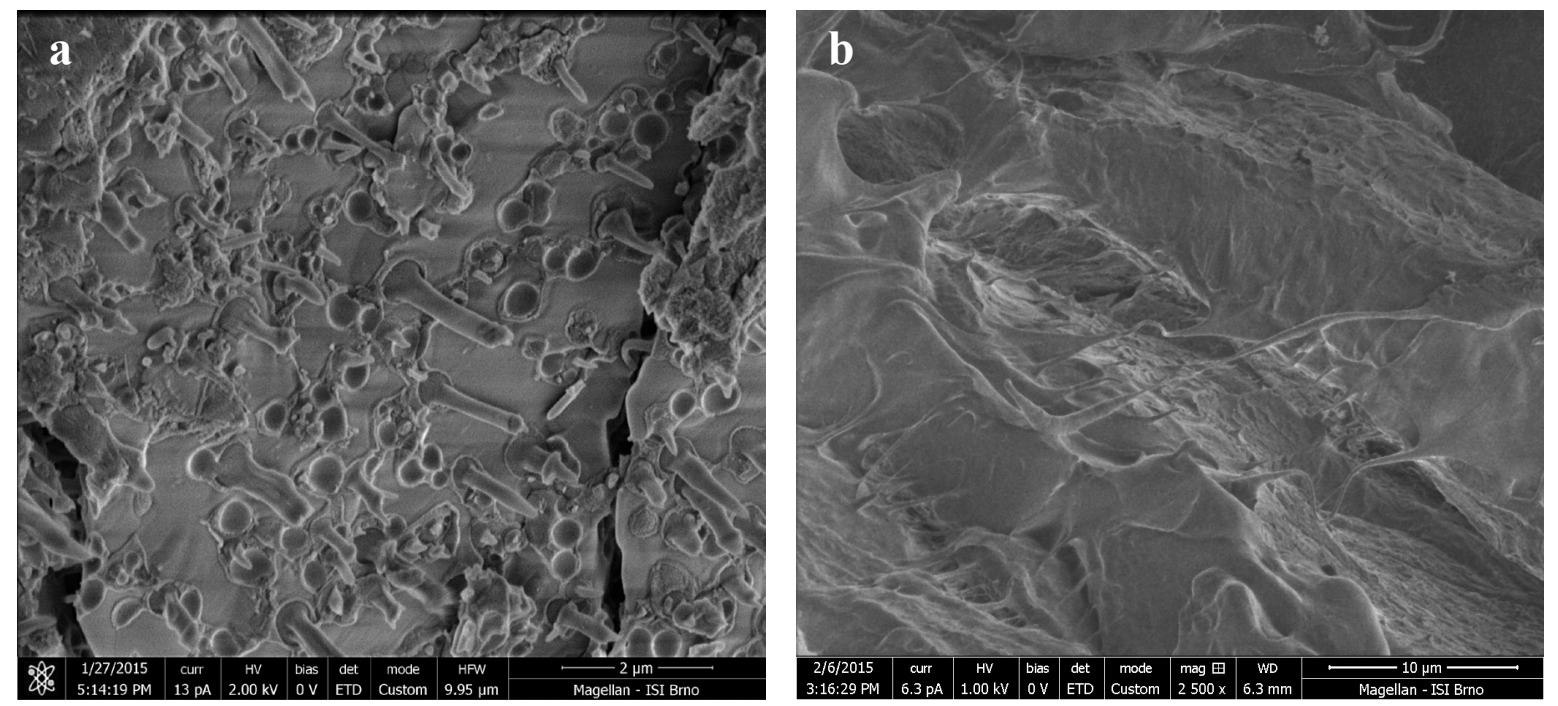

Figure 1. a). Cryo-SEM image of Cupriavidus necator H16. Needle-type deformations can be clearly seen within the fractured sample surface. b) Cryo-SEM image of pure sample of $\mathrm{P}(\mathrm{HB}-\mathrm{co}-\mathrm{HV})$. This sample shows needle-type deformation.

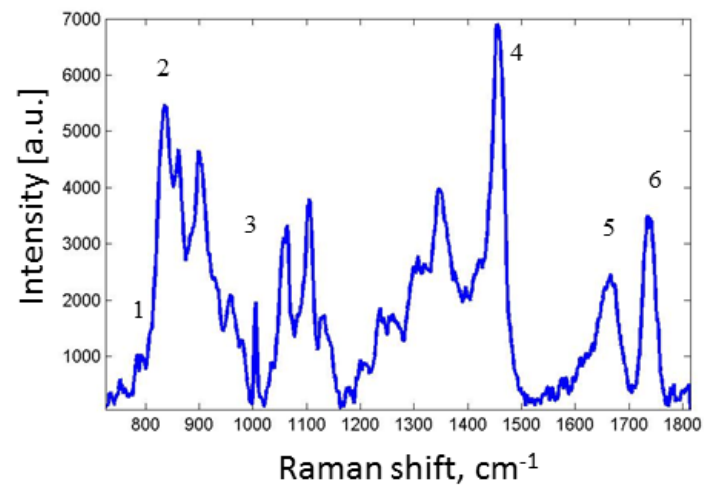

\begin{tabular}{|l|l|l|}
\hline Peak No. & $\begin{array}{l}\text { Raman feature, } \\
\text { cm }^{-1}\end{array}$ & $\begin{array}{l}\text { Suggested } \\
\text { assignment }\end{array}$ \\
\hline 1 & 786 & DNA \\
\hline 2 & 837 & PHB \\
\hline 3 & 1005 & Phenylalanine \\
\hline 4 & 1455 & PHB \\
\hline 5 & 1662 & Amide I, lipids \\
\hline 6 & 1736 & PHB \\
\hline
\end{tabular}

Figure 2. Raman spectra of Cupriavidus necator H16. The most prominent contributions are collected in the Table. 